'Grupo "GRINMADE", Facultad de Medicina, Universidad de Antioquia. Colombia.

${ }^{2}$ Grupo "Comunicación

Humana", Departamento de Fonoaudiología, Facultad de Salud, Universidad de Pamplona, Colombia.

Trabajo no recibió financiamiento. Los autores declaran no tener conflictos de interés.

Recibido el 5 de septiembre de 2017, aceptado el 10 de septiembre de 2018.

Correspondencia a: Javier Martínez Torres Facultad de Medicina, Universidad de Antioquia. Colombia. epid_javier@hotmail.com

\section{Prevalencia de vida y factores asociados al consumo de marihuana en estudiantes escolarizados de Pamplona-Colombia, durante el primer período de 2015: estudio Emtamplona}

\author{
JAVIER MARTÍNEZ-TORRES ${ }^{1}$, HERIBERTO RANGEL-NAVIA ${ }^{2}$, \\ ELIANA RIVERA-CAPACHO ${ }^{2}$
}

\section{Marijuana consumption among Colombian adolescents}

Background: Marijuana consumption is a public health problem. Aim: To determine the life time prevalence of marijuana use among Colombian adolescents and the factors associated with its consumption. Material and Methods: Secondary analysis of data from a study aimed to determine the prevalence of smoking. A self-administered and anonymous questionnaire was answered by 814 adolescents aged $14 \pm 2$ years ( 439 women). Results: Ten percent of respondents consumed marijuana at least once in their life. A logistic regression analysis showed that professing a non-christian religion, being smoker and having an age over 12 years, was associated with marijuana consumption. Conclusions: There is a high frequency of marijuana use among these adolescents.

(Rev Med Chile 2018; 146: 1016-1023)

Key words: Adolescent; Colombia; Cross-Sectional Studies; Marijuana Smoking.
L a adolescencia es un ciclo clave durante el que se producen una serie de cambios psicosociales y físicos ${ }^{1}$, desde el punto de vista neurobiológico social, es una etapa importante, pues en ella se inician relaciones seudoindependientes con otros seres humanos, diferentes a sus compañeros de colegio, familiares y vecinos²; la relación con sus pares es vital para el desarrollo de aptitudes sociales, de hecho la amistad se convierte en lo más importante y relega el apego que se tenía hasta entonces por los padres ${ }^{1}$. Los adolescentes muestran la tendencia a compartir sus posiciones (sociales, musicales, religiosas) con sus pares, generalmente solo con aquellos que piensan de manera similar's.

La marihuana es una sustancia ampliamente utilizada en la sociedad, especialmente entre los adolescentes ${ }^{4}$. Su consumo se ha convertido en un problema de salud pública ${ }^{5}$, pues genera dependencia y su prevalencia de vida en Colombia ha aumentado en los últimos años ${ }^{6}$; entre los efectos deletéreos de su uso se encuentran cambios fisiopatológicos, tales como alteraciones inflamatorias y lesiones precancerosas de las vías respiratorias, relacionada proporcionalmente con la dosis que se consume $e^{7}$; en el campo psicológico social se encuentran el deterioro cognitivo, con mayor énfasis en memoria y aprendizaje, así como la pérdida del control interno ${ }^{8}$, alteración en la función social ${ }^{9}$, trastornos afectivos, de ansiedad, de conducta, de la conducta alimentaria ${ }^{10}$.

El reporte de drogas muestra que la prevalencia 
de consumo de marihuana en adolescentes escolarizados aumentó de 7,59\%, en el año 2004, a 8,28\% en el año 2011; ; por otra parte, el Estudio Nacional de Consumo de Sustancias Psicoactivas ${ }^{11}$, desarrollado en el año 2013, muestra que 4,35\% (IC95\% 3,43\%-5,27\%) de los adolescentes entre 12 y 17 años han consumido marihuana durante el último año; en la ciudad de Bucaramanga se encontró, en estudiantes de décimo y undécimo grado, que 5,5\% de los adolescentes habían consumido marihuana al menos una vez en la vida, $y$ $4,4 \%$ lo había hecho durante el último año ${ }^{12}$. Por lo expuesto, y la falta de datos sobre el consumo de esta sustancia en la ciudad de Pamplona, aparece la necesidad de conocer el comportamiento de consumo local de esa sustancia.

Tradicionalmente se ha estudiado la prevalencia en el último mes y en los últimos doce meses, pues son marcadores robustos del consumo actual ${ }^{13}$, no obstante, la prevalencia de vida es un indicador que muestra el uso exploratorio de esta sustancia y, a su vez, permite evaluar la proporción de uso frecuente cuando se realiza evaluación de tendencias en el consumo de marihuana. Por ende, el objetivo de este trabajo es examinar la prevalencia de vida y factores sociodemográficos asociados al consumo de marihuana, en una muestra representativa de estudiantes de secundaria en Pamplona, Norte de Santander, Colombia.

\section{Materiales y Métodos}

\section{Tipo de estudio y población}

Estudio descriptivo transversal. Es un análisis secundario del estudio Emtamplona -Emtajoven Pamplona-, que buscó estimar la prevalencia de consumo de cigarrillo de tabaco entre adolescentes escolarizados, en el año 2015 en Pamplona, Colombia. La población y los supuestos para el cálculo del tamaño muestral han sido previamente reportados ${ }^{14}$. Los supuestos son suficientes para estimar la prevalencia del consumo de marihuana en la vida.

\section{Procesos de medición y recolección de la información}

La recolección de la información se realizó tomando como base la Emtajoven ${ }^{15}$. Encuesta escolar diseñada para evaluar el consumo de tabaco entre los jóvenes y ha sido aplicada alrededor del mundo, es un instrumento estructurado, autodiligenciado y anónimo, de aplicación individual. Durante la recolección de datos, los participantes recibieron explicación sobre la importancia de la medición, así como todas las pautas necesarias para el correcto diligenciamiento de la encuesta. El grupo encuestador siempre estuvo disponible y al pendiente de las inquietudes que manifestaban los adolescentes frente al cuestionario. Se hizo una prueba piloto con 40 sujetos de diferentes cursos académicos.

\section{Consumo de marihuana}

Para evaluar el consumo de marihuana se usó la pregunta: ¿Ha consumido marihuana alguna vez en la vida, aunque sea una o dos aspiraciones? El adolescente, al responder, marcaba un sí o un no.

\section{Factores sociodemográficos}

En el presente estudio se definieron como factores asociados las siguientes variables sociodemográficas: 1) sexo (masculino y femenino); 2) curso actual (sexto-séptimo, octavo-noveno, décimo-undécimo); 3) edad (10-11 años; 1213 años; 14-15 años; 16-17 años y 18-19 años); 4) tipo de colegio (público y privado); 5) religión (cristiano católico, cristiano no católico y ateo/ otros); 6) estrato social (a. Bajo-bajo, b. Bajo y Medio-bajo, y c. Medio-Medio-Alto) ${ }^{16}$; 7) grado académico de la madre u 8 ) del padre (no tiene padre/madre/no sabe, sin educación, hasta bachillerato, estudios superiores); 9) consumo actual de cigarrillo (sí o no). La definición de fumador ha sido reportado previamente ${ }^{14}$.

\section{Análisis estadístico}

En primer lugar, se realizó un análisis exploratorio para determinar la distribución de frecuencias (medidas de tendencia central y de dispersión para variables cuantitativas), frecuencias relativas (para variables cualitativas). Se realizó la descripción para la prevalencia de vida por variables sociodemográficas. Después de eso, para estimar la asociación entre el consumo de vida con las variables sociodemográficas de los adolescentes, se utilizaron dos modelos de regresión logística binaria $^{17}$.

El primer modelo de regresión logística binaria fue simple (odds ratio [OR] no ajustado), el segundo modelo fue multivariable (modelo $1^{\text {a }} \mathrm{OR}$ ). Se realizó ajuste para por edad, sexo y religión, esto 
debido a que en nuestro modelo teórico, estas variables son aquellas que ejercen un potencial efecto confusor, no se incluyó el curso (a pesar de ser significativo) debido a que este tiene una fuerte relación con la edad y no aportaba al modelo. Los análisis fueron realizados en SPSS versión 20, se consideró como significante un valor $\mathrm{p}<0,05$, la estimación de parámetros se hizo con un nivel de confianza de 95,0\%.

\section{Consideraciones éticas}

El estudio se desarrolló de acuerdo con las normas establecidas en la Resolución 8.430 de 1993, promulgada por el Ministerio de Salud de Colombia; de acuerdo con su Artículo 11, esta investigación se clasifica como «sin riesgo». La participación fue voluntaria, los tutores legales de los adolescentes firmaron el consentimiento informado y los adolescentes firmaron el asentimiento informado. El estudio fue aprobado por el Comité de Ética de la Universidad del Norte, acta de evaluación número 125 del 30 de abril de 2015.

\section{Resultados}

Se repartieron 1.000 consentimientos y asentimientos informados, en 138 casos los padres o adolescentes decidieron no ser parte de la investigación (13,8\% porcentaje de no participación), de los 862 adolescentes evaluados se analizaron 814, que completaron totalmente las preguntas relacionadas con el consumo de marihuana $(94,4 \%$ porcentaje de respuesta). La edad de los incluidos fue de 13,92 años DE 1,90 (IC95\% 13,79-14,05). La prevalencia de vida de consumo de marihuana fue de 10,2\% (83 adolescentes). Aquellas que acusaron consumir tabaco, pertenecer a la religión 'otros' y quienes tenían 18-19 años, mostraron las mayores prevalencias de vida $(34,9 \%, 21,9 \%$ y $39,0 \%)$, respectivamente. Los demás datos se muestran en la Tabla 1.

La Figura 1 muestra que, tras realizarse el ajuste multivariable, se encontró que pertenecer a una religión diferente a la cristiana (OR 2,88 IC95\% 1,16-7,13), ser consumidor de tabaco (OR 6,20 IC95\% 3,69-10,42) y tener entre 14 y 15 años (OR 3,42 IC95\% 1,01-11,64), 16 y 17 años (OR 7,68 IC95\% 2,27-26,00), 18 y 19 años (OR 8,92 IC95\% 1,95-40,93) se asoció con el consumo de marihuana en la vida.

\section{Discusión}

Este es el primer estudio que examina el consumo de marihuana en una muestra significativa de adolescentes escolarizados en Norte de Santander, Colombia. Se observó una prevalencia reportada de consumo de marihuana en la vida de $10,2 \%$, en los adolescentes encuestados, cifra considerable en términos de salud pública; en el año 2013, el informe del Estudio Nacional de Consumo de Sustancias Psicoactivas encontró que $13,0 \%$ de los colombianos (entre 12 y 65 años) reportaron haber consumido en la vida al menos una sustancia psicoactiva ${ }^{11}$. Por otro lado, el Estudio Nacional de Consumo de Sustancias Psicoactivas, en población escolar de 2011, mostró que $7,0 \%$ de los escolares en Colombia declararon haber fumado marihuana al menos una vez en la vida $^{18}$. En Montería, Colombia, en el año 2013, se encontró que el consumo de vida fue de $12,1 \%$ en estudiantes entre 13 y 18 años $^{19}$; recientemente, en Chicoral, Tolima, en el año 2012, se encontró que 4,9\% de los adolescentes entre 8 y 17 años habían consumido marihuana al menos una vez en la $v_{\text {vida }}{ }^{20}$. En Pamplona, en el año 2013, estudiantes universitarios entre 18 y 25 años de edad acusaron que $25 \%$ de ellos habían consumido marihuana alguna vez en su vida, y alrededor de 7\% la había consumido durante el último mes ${ }^{21}$. El reporte mundial de drogas muestra que las prevalencias de vida en Argentina para el 2014, en adolescentes de 14-15 años fue de 18,3\%, en Chile para el 2015 en adolescentes de 15-16 años, fue de 43,6\%; en Venezuela, para el 2011, en adolescentes de 15-17 años, fue de $3,2 \%{ }^{22}$.

En América Latina se han reportado diferentes prevalencias de vida. En Perú, en el año 2014, se encontró una prevalencia de vida de $9,1 \%{ }^{23}$, mientras que en Costa Rica, en el año 2012, se reportó $15,1 \%{ }^{24}$. Entre tanto, en Chile, en el año 2014, el décimo estudio nacional de drogas en población escolar mostró que la prevalencia fue de $37,2 \%{ }^{25}$. Otro estudio desarrollado en Chile mostró que el 14,5\% de los estudiantes del área Metropolitana de Santiago aseguraron haber consumido marihuana al menos cuatro veces durante el último $\mathrm{mes}^{26}$. En México se halló que 7,2\% de mujeres adolescentes de Veracruz habían consumido marihuana ${ }^{27}$. En Argentina, en el año 2005, se encontró que el consumo se había dado en 15,6\% de los adolescentes ${ }^{28}$. En países europeos, varios estudios han descrito 
Tabla 1. Prevalencia de vida de consumo de de marihuana por variables sociodemográficas

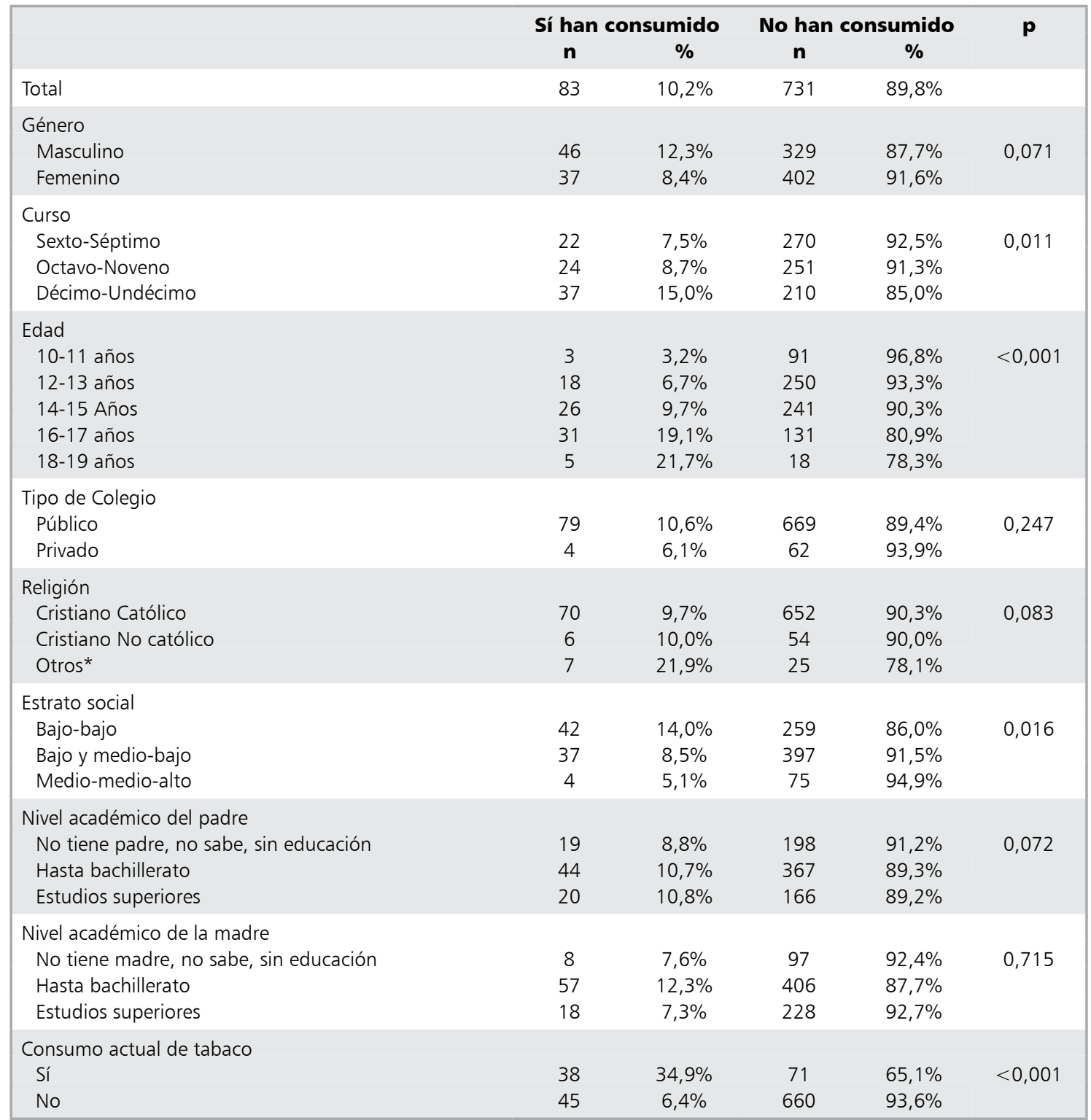

*Incluye ateo y personas que aseveraron ser de otras religiones. Valor p calculado con $\chi^{2}$.

diferentes prevalencias de vida en adolescentes, como los documentados en República Checa, durante el año 2014 , fue $23,1 \%{ }^{29}$, y en Islandia, en el año 2015, fue de $8,0 \%{ }^{30}$.

La prevalencia por sexo mostró que $12,3 \%$ de los hombres y $8,4 \%$ de las mujeres reportaron haber fumado marihuana, estos resultados son superiores a los hallados en el estudio nacional de sustancias psicoactivas en el año 2011, en el que se reportó una prevalencia mayor para el sexo masculino $(8,6 \% \text { vs } 5,5 \%)^{18}$. Estudios desarrollados en Costa Rica ${ }^{24}$ y Chile ${ }^{25,26}$ han mostrado una tendencia similar. Un estudio previo realizado en Pamplona, con población universitaria, mostró que los hombres eran quienes tenían una mayor prevalencia de vida $(25,9 \% \text { vs } 15,6 \%)^{21}$. Por otro 


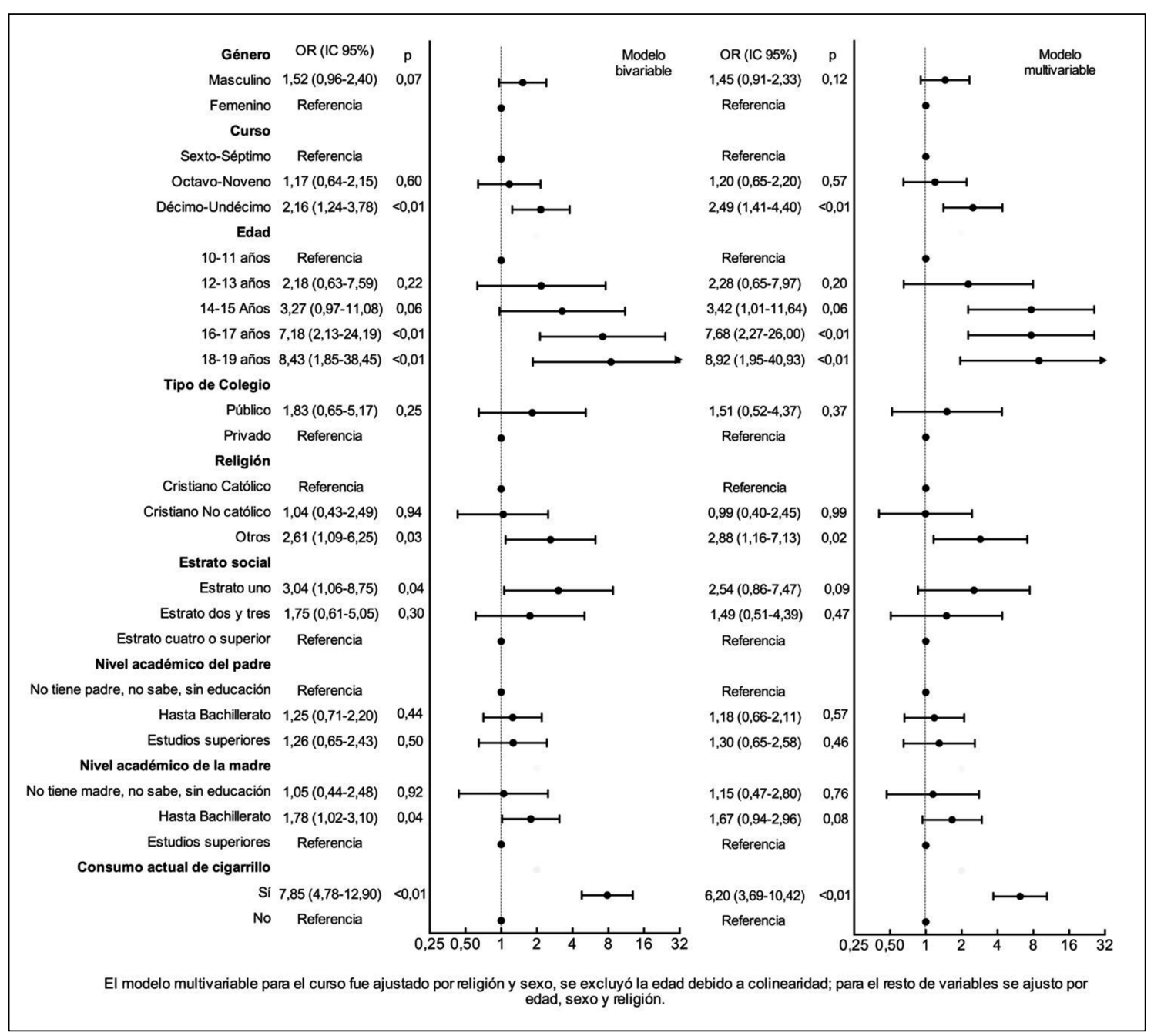

Figura 1. Asociación entre consumo marihuana en la vida y variables sociodemográficas.

lado, en países europeos, como República Checa, en el año $2014^{29}$, se observó que las prevalencias de vida no muestran diferencias por sexo (femenino $23,3 \%$ vs masculino $22,8 \%$ ); este resultado es similar al reportado por Armason et $\mathrm{al}^{30}$ en adolescentes de Islandia, en el año 2015 (8,0\% versus $8,1 \%$ ).

Las creencias religiosas determinan estilos de vida y comportamientos de los individuos ${ }^{31}$. En este estudio se encontró que aquellos que confesaron pertenecer a una creencia religiosa diferente al cristianismo mostraron una prevalencia de $21,9 \%$, la cual es dos veces superior a la de los cristianos; resultado que concuerda por Chen CY et $\mathrm{a}^{32}$, quienes describieron que en adolescentes de Sudamérica, aquellos que tenían niveles más altos de práctica religiosa están inversamente asociados con las primeras etapas de experiencias con cannabis, en la misma línea, un metaanálisis con 22 estudios desarrollados entre 1995 y 2007 mostró el mismo comportamiento ${ }^{33}$.

A medida que los cursos escolares son superiores, de manera proporcional se reportan prevalencias más altas, resultados que concuerdan con los hallados en Costa Rica en el año $2015^{24}$; no obstante, cabe resaltar que este puede ser un factor que no tiene relación directa con el consumo de marihuana, pues en el análisis multivariable se 
encontró que dicha asociación perdía su efecto. Un estudio desarrollado en la ciudad de Bucaramanga reportó una prevalencia menor a la hallada en el presente estudio entre estudiantes de décimo y undécimo, donde se encontró que 5,5\% de ellos habían consumido marihuana al menos una vez en la vida ${ }^{12}$, en comparación con 15,5\% hallado en el presente estudio.

Tradicionalmente, el estrato socioeconómico se ha descrito como un determinante del comportamiento y de la salud de la población ${ }^{34}$. En el presente análisis se halló que los estudiantes de estratos bajos presentan mayor prevalencia que sus similares de estratos altos $(14,0 \%$ vs $5,1 \%)$, resultado que concuerda por el reportado en una muestra de adolescentes chilenos ${ }^{26}$ y en estudiantes universitarios de Pamplona, Colombia ${ }^{21}$. Adicionalmente, esta proporción es análoga a la prevalencia de consumo de tabaco reportada en esa misma población, previamente ${ }^{14}$. El estrato social está ligado al tipo de colegio al que los jóvenes asisten; es así que los individuos que asisten a colegios públicos presentan una mayor prevalencia $(10,6 \%$ vs $6,1 \%$ ), hallazgo que va en la misma línea que el reportado por Luchese $\mathrm{M}$ et $\mathrm{al}^{35}$ en estudiantes de 11 a 12 años, de Córdoba, Argentina, Serfaty et $\mathrm{al}^{28}$ en Buenos Aires y Morales et a ${ }^{36}$ en Barcelona, España; no obstante, difieren de un estudio peruano, desarrollado en el año 2008, en el que se reportó que no existían diferencias relacionadas con el tipo de institución educativa ${ }^{37}$.

Aquellos que confiesan consumir tabaco mostraron la prevalencia más alta, con 34,9\%; tal resultado concuerda con lo descrito por Iglesias et $\mathrm{al}^{38}$, quienes aseveran que el cigarrillo de tabaco es una precuela para el consumo de marihuana. Este comportamiento es análogo al que sucede con el uso del narguile en adolescentes, pues un estudio canadiense describió que los sujetos que consumen cigarrillo tradicional eran los que presentaban mayor consumo de marihuana ${ }^{39}$.

En conclusión: la prevalencia de vida del consumo de marihuana es alta en la población estudiada. Esos datos podrían utilizarse para ofrecer programas de intervención educativa acerca de los riesgos del consumo de dichas sustancias.

\section{Limitaciones}

Los autores declaramos posibles sesgos de selección, pues hubo 13,8\% de los invitados rechazaron ser parte de la investigación, y 5,6\% de los que aceptaron no respondieron las preguntas; con respecto a sesgos de clasificación: algunos adolescentes que han consumido marihuana pudieron haber reportado este componente de manera negativa, por otro lado, a pesar de ser un análisis secundario del proyecto Emtamplona ${ }^{14}$, el tamaño de la muestra es lo suficientemente grande para estimar la prevalencia, pero no es lo suficientemente potente estimar asociaciones con errores tipo II menores a $20 \%$, se aclara que en todas las asociaciones la potencia es inferior a $80 \%$.

\section{Referencias}

1. Iglesias Díaz J. Desarrollo del adolescente: aspectos físicos, psicológicos y sociales. Pediatr Integral 2013; 17 (2): 88-93.

2. Burnett S, Sebastian C, Cohen Kadosh K, Blakemore S. The social brain in adolescence: evidence from functional magnetic resonance imaging and behavioural studies. Neurosci Biobehav Rev 2011; 35 (8): 1654-64.

3. Piaget J, Inhelder B. El pensamiento del adolescente. En: De la lógica del niño a la lógica del adolescente. Barcelona, España. Paidos; 1985.

4. Torres G, Fiestas F. Efectos de la marihuana en la cognición: una revisión desde la perspectiva neurobiológica. Rev Peru Med Exp Salud Publica 2012; 29 (1): 127-34.

5. Quimbayo-Díaz J, Olivella-Fernández M. Consumo de marihuana en estudiantes de una universidad colombiana. Rev Salud Pública 2013; 15 (1): 32-43.

6. Observatorio de drogas de Colombia, Ministerio de Justicia y del Derecho. Reporte de Drogas de Colombia. Bogota DC. 2016. http://www.odc.gov.co/Portals/1/publicaciones/pdf/odc-libro-blanco/ODC0100322016_reporte_drogas_colombia_2016.pdf.

7. Kalant H. Adverse effects of cannabis on health: an update of the literature since 1996. Prog Neuropsychopharmacol Biol Psychiatry 2004; 28 (5): 849-63.

8. Vélez García A, Ostrosky-Solís F, Borja Jiménez K. Efectos del consumo de marihuana sobre la toma de decisión. Revista Mexicana de Psicología 2010; 27 (2): 309-15.

9. Kohn L, Kittel F, Piette D. Peer, family integration and other determinants of cannabis use among teenagers. Int J Adolesc Med Health 2004; 16 (4): 359-70.

10. Moore T, Zammit S, Lingford-Hughes A, Barnes T, Jones $\mathrm{P}$, Burke $\mathrm{M}$, et al. Cannabis use and risk of psychotic or affective mental health outcomes: a systematic review. Lancet 2007; 370 (9584): 319-28. 
11. Ministerio de Justicia y del Derecho, Ministerio de Salud y proteccion social. Estudio Nacional de Consumo de Sustancias Psicoactivas en Colombia. Bogotá DC. 2013. https://www.unodc.org/documents/colombia/2014/ Julio/Estudio_de_Consumo_UNODC.pdf.

12. Martínez-Mantilla J, Amaya-Naranjo W, Campillo H, Rueda-Jaimes G, Campo-Arias A, Díaz-Martínez L. Consumo de Sustancias Psicoactivas en Adolescentes, Bucaramanga, Colombia, 1996-2004. Rev Salud Pública 2007; 9 (2): 215-29.

13. Hynes M, Demarco M, Araneda J, Cumsille F. Prevalence of Marijuana Use among University Students in Bolivia, Colombia, Ecuador, and Peru. Int J Environ Res Public Health 2015; 12 (5): 5233-40.

14. Martínez-Torres J, Peñuela Epalza M. Prevalencia y factores asociados al consumo de cigarrillo tradicional, en adolescentes escolarizados. Rev Med Chile 2017; 145 (3): 309-18.

15. World Health Association. Tobacco Free Initiative (TFI). [Online]; 2010. (Acceso el 28 de septiembre de 2014). Disponible en: http://www.who.int/tobacco/ surveillance/gyts/en/\#.

16. Departamento Administrativo Nacional de Estadisticas. [Online]; 2016. Disponible en: https://www.dane.gov. co/files/geoestadistica/Preguntas_frecuentes_estratificacion.pdf.

17. Cerda J, Vera C, Rada G. Odds ratio: aspectos teóricos y prácticos. Rev Med Chile 2013; 141 (10): 1329-35.

18. Ministerio de Justicia y del Derecho, Ministerio de Salud y Protección Social. Estudio Nacional de Consumo de Sustancias Psicoactivas en Colombia en población escolar, Bogotá DC, 2011. https://www.unodc.org/documents/colombia/Documentostecnicos/Estudio_Consumo_Escolares.pdf.

19. Guerrero-Martelo M, Gonzalo Galván G, Pinedo-López J, Vásquez-De la Hoz F, Torres-Hoyos F, Torres-Oviedo J. Prevalencia de vida de uso de cannabis y rendimiento académico en adolescentes. Salud Uninorte 2015; 31 (3): 467-78.

20. Aguirre-Guiza N, Aldana-Pinzón O, Bonilla-Ibáñez C. Factores familiares de riesgo de consumo de sustancias psicoactivas en estudiantes de una institución de educación media técnica de Colombia. Rev Salud Pública 2017; 19 (1): 3-9.

21. Martínez Torres J, Arias Coronel F, Rodelo-Vega A, Jaraba-Toro N, Meza-Castellanos L, Contreras-Jáuregui $\mathrm{M}$, et al. Prevalencia y factores asociados al consumo de marihuana en estudiantes de 18 a 25 años de una universidad pública, Colombia. Rev Univ Sal 2016; 18 (3): 525-31.

22. Oficina de las Naciones Unidas para las drogas y el deli- to. Organización de las naciones unidas. [Online]; 2018. (Acceso el 16 de junio de 2018). Disponible en: https:// dataunodc.un.org/drugs/prevalence_youth.

23. Bueno Castro L, Guerrero Vargas J, Pedrajas R, Tam Phun E. Prevalencia de consumo de marihuana en estudiantes de secundaria. Rev Enferm Herediana 2015; 8 (1): 17-23.

24. Instituto sobre Alcoholismo y Farmacodependencia. Área Desarrollo Institucional. Proceso de Investigación. IV Encuesta Nacional sobre Consumo de Drogas en Población de Educación Secundaria. Costa Rica: San José de Costa Rica, 2015. https://www.iafa.go.cr/ investigaciones/304-iv-encuesta-nacional-sobre-consumo-de-drogas-en-poblacion-de-educacion-secundaria.

25. Observatorio Chileno de Droga, Décimo estudio nacional de drogas en población escolar. Santiago: Servicio Nacional para la Prevención y Rehabilitación del Consumo de Drogas y Alcohol (SENDA), Santiago de Chile 2013. http://www.senda.gob.cl/wp-content/ uploads/2014/07/decimo-escolares-2013.pdf.

26. Dörr A, Gorostegui M, Viani S, Dörr B M. Adolescentes consumidores de marihuana: implicaciones para la familia y la escuela. Salud Ment 2009; 32 (4): 269-78.

27. Cortaza Ramírez L, Villar LM, Puig Nolasco A. Consumo de drogas en adolescentes mexicanas. Revista Iberoamericana De Educación E Investigación En Enfermería 2012; 2 (3): 19-25.

28. Serfaty E, Andrade J, Ortiz Z, Masaútis A, Negri G. Descripción del perfil de consumo de sustancias psicoactivas en población adolescente de 15 a 18 años del Conurbano Bonaerense. Bol ANM 2006; 2: 317-30.

29. Kážmér L, Csémy L, Ružbarská I, Pavelka J, Hamřík Z, Kalman M. Trends in Lifetime Cannabis Use among Czech School-aged Children from 2002 to 2014. Cent Eur J Public Health 2017; 25 (Supl 1): S47-51.

30. Arnarsson A, Kristofersson G, Bjarnason T. Adolescent alcohol and cannabis use in Iceland 1995-2015. Drug Alcohol Rev 2018; 37 (Suppl 1): S49-S57.

31. Turner-Musa J, Lipscomb L. Spirituality and social support on health behaviors of African American undergraduates. American Journal of Health Behavior. 2007; 31 (5): 495-501.

32. Chen C, Dormitzer C, Bejarano J, Anthony J. Religiosity and the earliest stages of adolescent drug involvement in seven countries of Latin America. Am J Epidemiol 2004; 159 (12): 1180-8.

33. Yeung J, Chan Y, Lee B. Youth religiosity and substance use: a meta-analysis from 1995 to 2007. Psychol Rep 2009; 105 (1): 255-66.

34. Betancour A, Zambrano R. Prevalencia del consumo de tabaco en una muestra de adolescentes escolarizados 
del municipio de Ebéjico, departamento de Antioquia, Colombia Liber 2014; 20 (1): 131-9.

35. Lucchese MS, Burrone MS, Enders JE, Fernández R. Consumo de sustancias y escuela: un estudio en adolescentes escolarizados de Córdoba. Revista de Salud Pública 2013; 17 (1): 32-41.

36. Morales E, Ariza C, NM, Péreza A, Sánchez F. Consumo de cannabis en los estudiantes de secundaria de Barcelona: inicio en el consumo, efectos experimentados y expectativas. Gac Sanit 2008; 22 (4): 321-9.

37. Gutiérrez C, Contreras H, Trujillo D, Haro E, Ramos-Castillo J. Drogas ilegales en escolares de Lima y Callao: factores familiares asociados a su consumo. Anales de la Facultad de Medicina 2009; 70 (4): 247 54.

38. Iglesias V, Cavada G, Silva C, Cáceres D. Consumo precoz de tabaco y alcohol como factores modificadores del riesgo de uso de marihuana. Rev Saúde Pública 2007; 41 (4): 517-22.

39. Minaker L, Shuh A, Burkhalter R, Manske S. Hookah use prevalence, predictors, and perceptions among Canadian youth: findings from the 2012/2013 Youth Smoking Survey. Cancer Causes Control 2015; 26 (6): 831-8. 